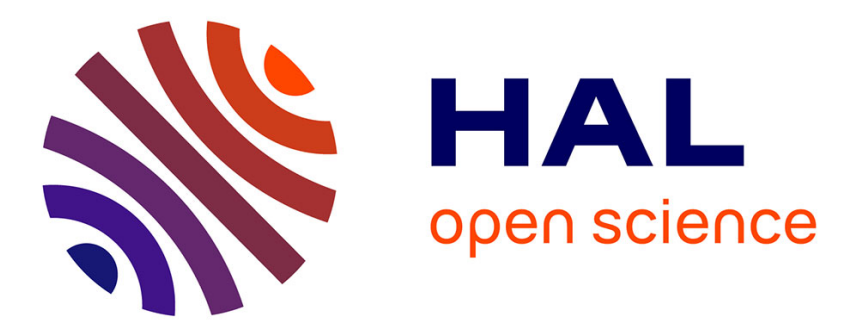

\title{
Blood vessel enhancement via multi-dictionary and sparse coding: Application to retinal vessel enhancing
}

Bin Chen, Yang Chen, Zhuhong Shao, Tong Tong, Limin Luo

\section{To cite this version:}

Bin Chen, Yang Chen, Zhuhong Shao, Tong Tong, Limin Luo. Blood vessel enhancement via multi-dictionary and sparse coding: Application to retinal vessel enhancing. Neurocomputing, 2016, 10.1016/j.neucom.2016.03.012 . hal-01331415

\section{HAL Id: hal-01331415 https://hal.science/hal-01331415}

Submitted on 13 Jun 2016

HAL is a multi-disciplinary open access archive for the deposit and dissemination of scientific research documents, whether they are published or not. The documents may come from teaching and research institutions in France or abroad, or from public or private research centers.
L'archive ouverte pluridisciplinaire HAL, est destinée au dépôt et à la diffusion de documents scientifiques de niveau recherche, publiés ou non, émanant des établissements d'enseignement et de recherche français ou étrangers, des laboratoires publics ou privés. 


\title{
Blood vessel enhancement via multi-dictionary and sparse coding: Application to retinal vessel enhancing
}

\author{
Bin Chen, ${ }^{\mathrm{a}, \mathrm{b}}$, Yang Chen ${ }^{\mathrm{a}, \mathrm{b}}$, Zhuhong Shao ${ }^{\mathrm{c}}$, Tong Tong ${ }^{\mathrm{d}}$, Limin Luo ${ }^{\mathrm{a}, \mathrm{b}}$ \\ a. Laboratory of Image Science and Technology, Southeast University, Nanjing, China \\ b. Centre de Recherche en Information Biomedicale Sino-Francais (LIA CRIBs), Rennes, France \\ c. College of Information Engineering, Capital Normal University, Beijing, China \\ d. Biomedical Image Analysis Group, Department of Computing, Imperial College London, London, UK
}

\begin{abstract}
Blood vessel images can provide considerable information of many diseases, which are widely used by ophthalmologists for disease diagnosis and surgical planning. In this paper, we propose a novel method for the blood Vessel Enhancement via Multi-dictionary and Sparse Coding (VE-MSC). In the proposed method, two dictionaries are utilized to gain the vascular structures and details, including the Representation Dictionary (RD) generated from the original vascular images and the Enhancement Dictionary (ED) extracted from the corresponding label images. The sparse coding technology is utilized to represent the original target vessel image with RD. After that, the enhanced target vessel image can be reconstructed using the obtained sparse coefficients and ED. The proposed method has been evaluated for the retinal vessel enhancement on the DRIVE and STARE databases. Experimental results indicate that the proposed method can not only effectively improve the image contrast but also enhance the retinal vascular structures and details.
\end{abstract}




\section{Keywords}

Blood vessel enhancement; Multi-dictionary; Sparse coding; Retinal vessel image

\section{Introduction}

The human body contains different types of blood vessels, which constitute a network of arteries and veins. The visualization of these blood vessels is important for disease diagnosis and improving the planning and navigation in interventional procedures [1-4]. For instance, retinal vessel images are widely used by ophthalmologists for the disease diagnosis such as diabetes, hypertension, cardiovascular disease and stroke. In case of changes in vessel caliber, branching angle or vessel tortuosity are results of hypertension [5]. The onset of neovascularization is a sign of diabetic retinopathy, and a complication of diabetes which leads to the cause of blindness [6]. However, due to the imperfect imaging condition, the quality of blood vessel images is usually poor, making it hard to recognize the vascular structure clearly. An effective way to overcome these issues is to use the image enhancement technology. The main purpose of blood vessel enhancement is to highlight the vascular structures and details [7-9]. In this paper, we propose a novel method for the blood Vessel Enhancement via Multi-dictionary and Sparse Coding (VE-MSC). The DRIVE ${ }^{1}$ and STARE$^{2}$ databases are used to evaluate the proposed method. Experimental results demonstrate that the image contrast is effectively improved and the retinal vessel structures and details are well enhanced. A comparison with state-of-the-art methods has also been carried out in the retinal vessel enhancement. 
The rest of the paper is organized as follows. In Section 2, the related works about blood vessel enhancement are briefly reviewed. The proposed method VE-MSC and its application on r e $\mathrm{t}$ i

$\mathrm{n}$ a

1. http://www.isi.uu.nl/Research/Databases/DRIVE/

2. http://www.ces.clemson.edu/ ahoover/stare/

vessel enhancement are presented in Section 3. In Section 4, the performance of retinal vessel enhancement is assessed by experiments on the DRIVE and STARE databases. Finally, a conclusion is given in Section 5.

\section{Related works}

According to different emphasis on the prior knowledge, blood vessel enhancement methods can be categorized into three groups: histogram based, transformation based and filter based.

(1) Histogram-based method [10-14] utilizes the prior information of blood vessel to equal the histogram distribution. For instance, histogram equalization (HE) [15] and contrast limited adaptive histogram equalization (CLAHE) [16] are two basic methods used for the retinal vessel enhancement. However, some retinal vessel details are lost during the equalization. Kuldeep et al. [17] proposed a contrast enhancement method based on the sub-image histogram equalization for low exposure gray scale image. The histogram was clipped using a threshold value as an average number of gray level occurrences to control the enhancement rate, but it was only used for the low exposure gray scale image.

(2) Transformation-based method transfers the image to other space [18-21], where the blood vessel can be enhanced. Miao et al. [22] proposed a retinal vessel enhancement algorithm based 
on multi-scale top-hat transformation and histogram fitting stretching. However, part of the vascular structures were changed through the transformation and histogram stretching. In addition, some parameters utilized in the method were sensitive to the initialization. Li et al. [23] applied the fractional Fourier transformation to the image enhancement. However, it also suffered the blood vessel details lost problem during the selection of image features.

(3) Filter-based method utilizes a filter or multiple filters [24-27] to enhance the blood vessel. Fraz et al. [28] proposed a retinal vessel enhancement method based on decision trees and Gabor filter, while it also had the vascular details lost problem. To overcome these weaknesses, a medical image enhancement method using morphology-based homo-morphic filtering technique was developed by Oh et al. [29]. However, the contrast of the blood vessel was low, and the vascular details lost problem was also existed.

The vascular details enhanced by these classical reported methods in literatures are hardly to be completely reserved, and the contrast of the enhanced vascular image is not high enough. To solve these problems, a novel blood vessel enhancement method using multi-dictionary and sparse coding is proposed in this paper. To the best of our knowledge, this is the first time to achieve the blood vessel enhancement via multi-dictionary and sparse coding. In order to gain the blood vascular structures and details, two corresponding dictionaries are generated. One is the representation dictionary (RD) generated from the blood vessel images, the other one is the enhancement dictionary (ED) extracted from the corresponding label images. The patches in RD and ED are selected through the information of the label images to optimize the multi-dictionary. Then the input target image is represented by RD to get the sparse coefficients via a sparse 
coding process. Finally, the enhanced blood vessel image is obtained from the solved sparse coefficients and ED. The effect of dictionary patch size and dictionary patch selection are analyzed in the experiments.

\section{Vessel Enhancement via Multi-dictionary and Sparse Coding (VE-MSC)}

\subsection{RD and ED generation}

Dictionary based methods have been studied in medical image processing [30-33], which differs in how they form the overcomplete dictionary. For instance, Chen et al. [34] proposed a Low-Dose CT (LDCT) image processing method based on artifact suppressed dictionary learning, where an overcomplete global dictionary was included. Li et al. [35] used the group-sparse representation with dictionary learning for medical image denoising and fusion, and the dictionary utilized was also overcomplete. In the proposed method, both the generated RD and ED are overcomplete, which are then used for enhancing the blood vascular structures and details.

Each patch in $\mathrm{RD}$ has the unique and corresponding patch in ED, and they have the same location in the respective dictionary. $\mathrm{RD}$ is generated from the original blood vessel images, ED is extracted from the corresponding label images with value 0 or 1 . Let $f$ be a $2 \mathrm{D}$ gray image in a blood vessel image database. The image size of $f$ is $M \times M$, and its sequence number in the blood vessel image database is $k$. Then a pixel with the location $(x, y)$ in $f$ can be remarked as $f(x, y, k)$. Let $f_{l}$ be the corresponding label image of $f$, which is the blood vessel segmentation result delineated manually by an expert. Then the corresponding label value 
of $f(x, y, k)$ in the label image $f_{l}$ is denoted as $f_{l}(x, y, k)$. A patch in $\mathrm{RD}\left(p^{\mathrm{RD}}\right)$ and its corresponding patch in ED ( $\left.p^{\mathrm{ED}}\right)$ are defined as:

$$
\begin{array}{ll}
p^{\mathrm{RD}}: f(\tau+s, \tau+s, k), \tau=-h,-h+1, \ldots h \quad \text { if } \sum_{\tau=-h}^{h} f_{l} \geq t \\
p^{\mathrm{ED}}: f_{l}(\tau+s, \tau+s, k), \tau=-h,-h+1, \ldots h \quad \text { if } \sum_{\tau=-h}^{h} f_{l} \geq t
\end{array}
$$

where $\forall s \in\{d, 2 d, 3 d, \ldots, m d\}, \forall k \in\{1,2, \ldots, L\}, h$ is the patch size, $d$ is a step value used to gain different patches, $m$ is the integer part of $M / d, L$ is the amount of blood vessel images in the database, and $t$ is a patch selection threshold value used to optimize the dictionaries. Fig .1 shows some patches in the RD and $\mathrm{ED}$ with $h=4, t=10$. The red boxes in

Fig .1 marked

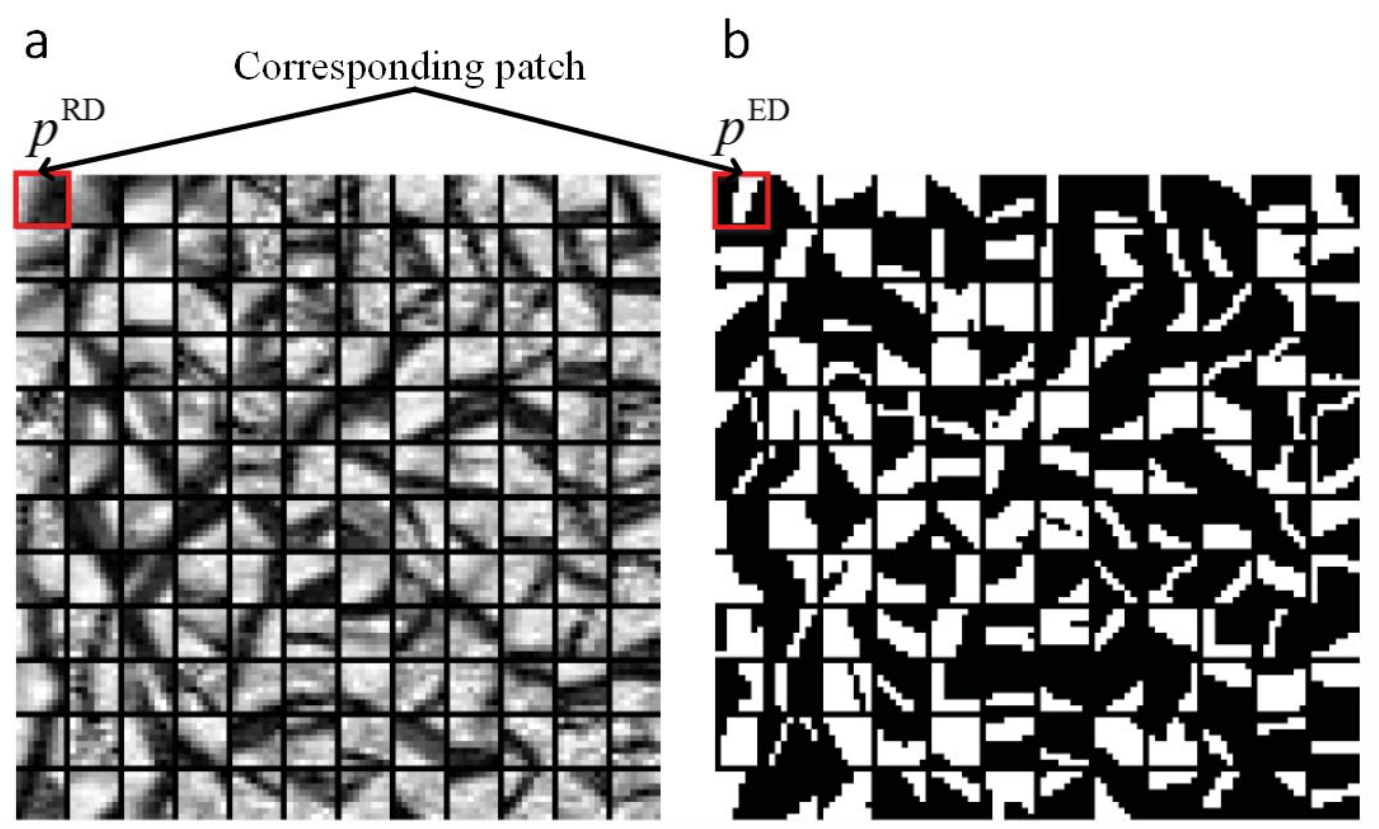

Fig. 1. Patches in the RD and $\mathrm{ED}, h=4, t=10$. (a) RD, the patches are extracted from the DRIVE training set, only use the green channel, (b) ED, the patches are extracted from the corresponding retinal vessel segmentation results (label images from the first expert).

the corresponding patches, they have the same location in the respective dictionary. Most patches in the dictionaries contain blood vessels due to the patch selection in Eq. (1). The impact of 
background is mostly eliminated through the ED, and the blood vascular structures and details can be mostly reserved by the two dictionaries.

\subsection{Sparse coding and blood vessel enhancement}

The multi-dictionary (RD and ED) is generated from Eq. (1), in order to describe the dictionaries clearly, let $p_{i}^{\mathrm{RD}}$ be the $i$-th patch in $\mathrm{RD}, \mathrm{RD}=\left[p_{1}^{\mathrm{RD}}, p_{2}^{\mathrm{RD}}, \ldots p_{n}^{\mathrm{RD}}\right]$, then $\mathrm{ED}$ $=\left[p_{1}^{\mathrm{ED}}, p_{2}^{\mathrm{ED}}, \ldots p_{n}^{\mathrm{ED}}\right]$. Let $f_{o}$ be the original blood vessel image to be enhanced (a $2 \mathrm{D}$ gray image), and $p_{f_{0}}$ is a patch extracted from $f_{0}$. To get the sparse coefficients used in the blood vessel enhancement processing, $p_{f_{0}}$ is represented as:

$$
p_{f_{0}}=\alpha_{1} p_{1}^{\mathrm{RD}}+\alpha_{2} p_{2}^{\mathrm{RD}}+\ldots+\alpha_{n} p_{n}^{\mathrm{RD}}
$$

Since the representation in Eq. (2) is sparse, most of the coefficient $\alpha_{i}$ will be zero, let $\alpha=\left[\alpha_{1}, \alpha_{2}, \ldots \alpha_{n}\right]$, and the $\alpha$ satisfy the restricted isometry property (RIP) [36, 37]. Then the sparse solution can be obtained by solving the following equation [38]:

$$
\hat{\alpha}=\min _{\alpha}\|\alpha\|_{0} \text { subject to }\left\|p_{f_{0}}-\mathrm{RD} \alpha\right\|_{2}^{2} \leq \varepsilon
$$

where the $\varepsilon$ is an error target for the sparse solution ( $\varepsilon=10^{-15}$ in our method), $l_{0}$-norm denotes the number of nonzero coefficients, and is the sparse constraint of this equation. Since $n \gg 2 h$, the Eq. (3) doesn't have a unique solution. However, when the solution of Eq. (3) is sparse enough, it can be solved efficiently by many sparse coding methods [39-41]. In the proposed method, an efficient Batch Orthogonal Matching Pursuit (Batch-OMP) method [42] is used for obtaining the sparse coding coefficients $\alpha$ in Eq. (3). Then we reconstruct the enhanced patch $p_{f_{e}}$ according to the corresponding relationship from RD to ED: 


$$
p_{f_{e}}=\alpha_{1} p_{1}^{\mathrm{ED}}+\alpha_{2} p_{2}^{\mathrm{ED}}+\ldots+\alpha_{n} p_{n}^{\mathrm{ED}}
$$

where $f_{e}$ is the enhanced blood vessel image made up of patches $p_{f_{e}}$, and the sparse coding coefficients $\alpha$ in Eq. (4) is the same as in Eq. (3). As the values in ED are binary, the gray value of $f_{e}$ is usually small. Then we rescale $f_{e}$ to $[0,255]$ by the following equation:

$$
f_{e r}=\frac{f_{e}-f_{e}^{\min }}{\max \left(f_{e}-f_{e}^{\min }\right)} \cdot 255
$$

where $f_{e}^{\min }$ is the minimum in $f_{e}, \max (\cdot)$ is an operator to find the maximum, and $f_{e r}$ is the final enhanced image.

The algorithm steps of the proposed VE-MSC are given as follows:

1) Gain RD and ED

a) Initial the $d, h, t, L$ in Eq. (1) and $\varepsilon$ in Eq. (3);

b) Extract the corresponding patch $p^{\mathrm{RD}}$ and $p^{\mathrm{ED}}$ using Eq. (1);

2) Represent the target image $f_{0}$ with $\mathrm{RD}$ (Eq. (2));

3) Solve the sparse coding coefficients $\alpha$ with Batch-OMP (Eq. (3));

4) Construct the enhanced patch $p_{f_{e}}$ using $\alpha$ and ED (Eq. (4));

5) Get the enhanced image $f_{e r}$ through a gray rescaling (Eq. (5)).

A detailed flow chart of the proposed method is shown in section 3.3 (application on retinal vessel enhancement). In general, the blood vessel image to be enhanced is not in the database used for the generation of RD and ED, but they are the same type of blood vessel images. For instance, we use the DRIVE to gain the dictionaries and the STARE to be the target image $f_{0}$ in the application of retinal vessel enhancement. 


\subsection{Retinal vessel enhancement via VE-MSC}

Fig. 2 shows a normal retinal vessel image and a background diabetic retinopathy retinal vessel

a

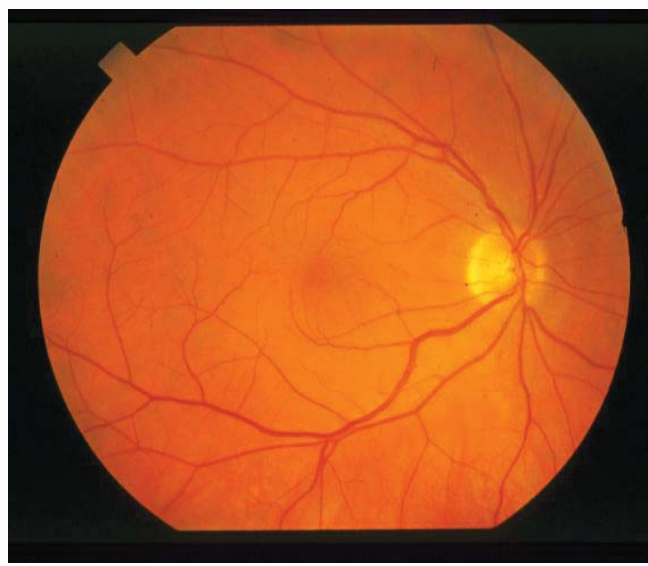

b

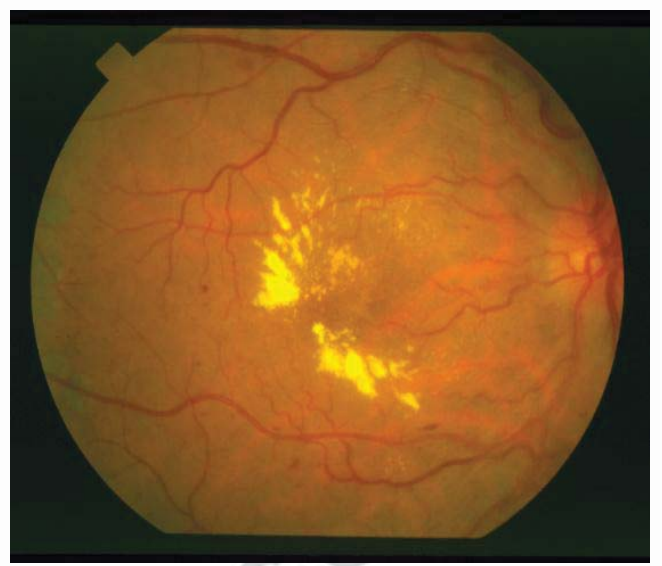

Fig. 2. Retinal vessel image. (a) Normal retinal vessel image in the STARE database, image index is 162, (b) Retinopathy retinal vessel image in the STARE database, image index is 1.

image. It can be observed that the retinal vascular structures and details are not clearly enough, therefore the proposed VE-MSC is utilized to overcome these issues. Hereinafter, only the green channel of the original color image is used as it offers the best vessel-background contrast [4, 9, 22]. Fig. 3 presents a flow chart of the retinal vessel enhancement method via VE-MSC. In order to enhance the image contrast and the retinal vascular structures and details, patches extracted 


\section{ACCEPTED MANUSCRIPT}

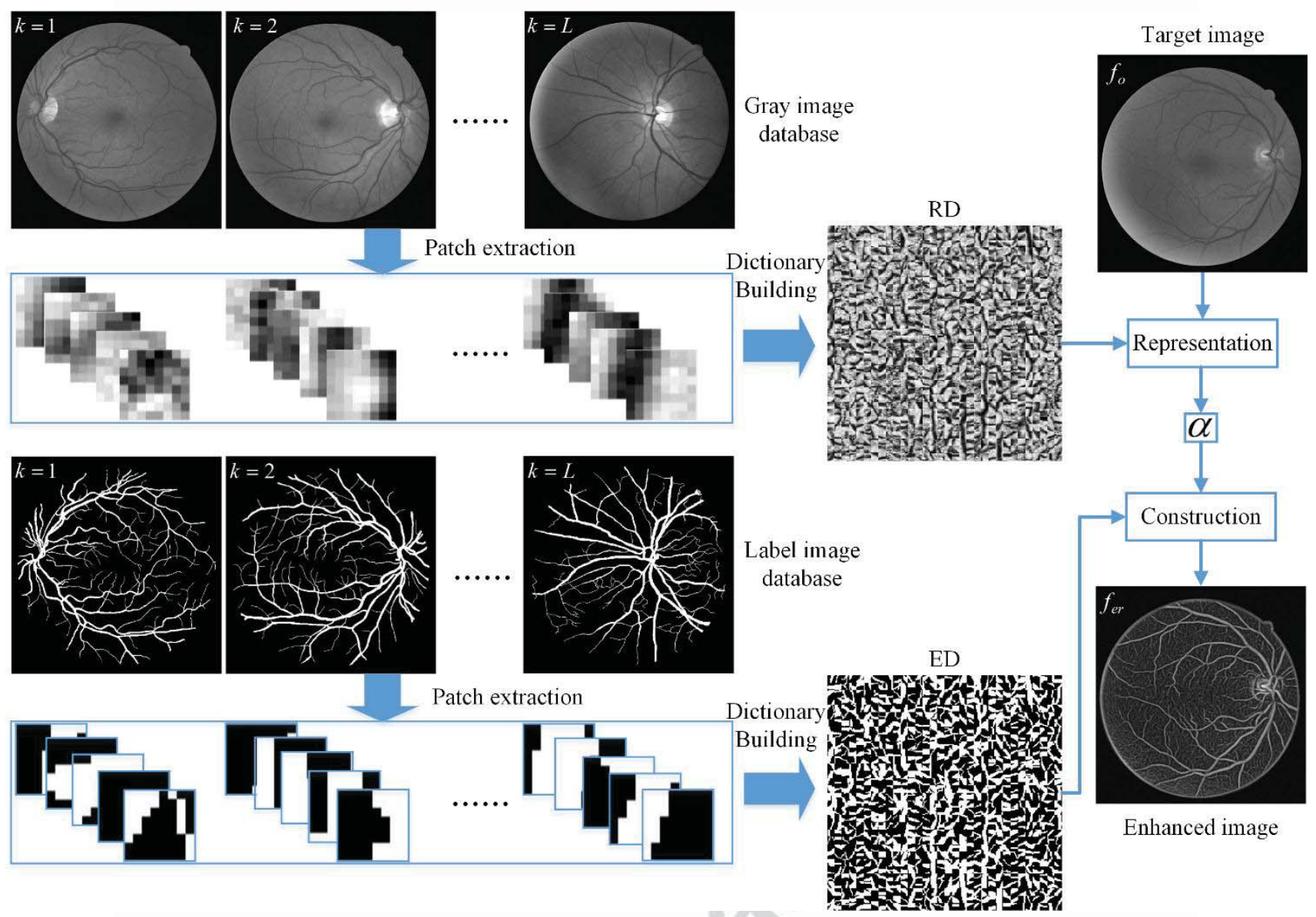

Fig. 3. Flow chart of the retinal vessel enhancement method via VE-MSC, $h=4, t=10$ (only use the green channel of the original RGB retinal image in the Gray image database).

from retinal vessel image database and label image database. The extracted patches ( $p^{\mathrm{RD}}$ and $\left.p^{\mathrm{ED}}\right)$ have the same location $(x, y, k)$ in the gray image database and label image database. The patches in RD have gray values, and the corresponding patches in ED have binary values. During the dictionary building process, most of the retinal vascular structures and details are obtained through a patch selection, which is introduced in Eq. (1). Then a representation to the target image $f_{o}$ is utilized to solve the sparse coefficients $\alpha$. After getting the dictionaries (in Eq. (1)) and sparse coefficients (in Eq. (3)), we use the ED and sparse coefficients to obtain the enhanced retinal vessel image with Eq. (4). Finally, the enhanced retinal vessel image is rescaled by Eq. 


\section{Experiments and results}

To evaluate the performance of the proposed method, a series of experiments are performed on the DRIVE and STARE databases. The DRIVE database consists of 40 color retinal images, which were captured by Canon CR5 nonmydriatic 3 Charge-Coupled-Device (CCD) cameras at $45^{\circ}$ field of view (FOV). Among them, the first 20 images are served as the testing set (image index No.1-20) and the rest constitute the training set (image index No.21-40), the size of each retinal image is $565 \times 584$ pixels. The STARE database contains 20 color retinal images (image index No.1-20, only use the images with hand labels) with the size of $700 \times 605$ pixels, which were captured by a TopCon TRV-50 fundus camera at $35^{\circ} \mathrm{FOV}$.

In order to evaluate the enhancement quality quantificationally, two metrics [29] are used:

(1) $C$ represents the contrast between the retinal vessels and the background (retinal regions except the vessels), which is defined as:

$$
C=\left|\frac{Y-G}{Y+G}\right|,
$$

where $Y$ is the average gray values of the retinal vessels, $G$ is the average gray values of the background.

(2) CII represents the contrast rate between enhanced image $\left(C_{e n}\right)$ and the original image $\left(C_{o r}\right)$, which is defined as:

$$
C I I=\frac{C_{e n}}{C_{o r}} .
$$

The larger the value of $C$ is, the more obvious the difference between the retinal vessels and the background is. It should be mentioned that black background pixels outside the pupil are not calculated in our method. In the experiment, the proposed approach was compared with HE [15], CLAHE [16], methods in [22, 28, 29]. Meanwhile, the effects of dictionary patch size $(h)$ and 
patch selection $(t)$ were also provided.

\subsection{Effect of dictionary patch size $(h)$}

The effect of using different patch size is analyzed in this section. Fig. 4 shows the $C$ values of DRIVE testing set with different patch sizes. In order to optimize the dictionaries (RD and ED), we set the parameter $t=\{5,10,14,18,25,36\}$, respectively. It can be observed that the best overall performance on the image contrast is achieved when the patch size is set to $12 \times 12$. The patch size $9 \times 9$ has the worst overall performance on the image contrast in Fig. 4 . This is because

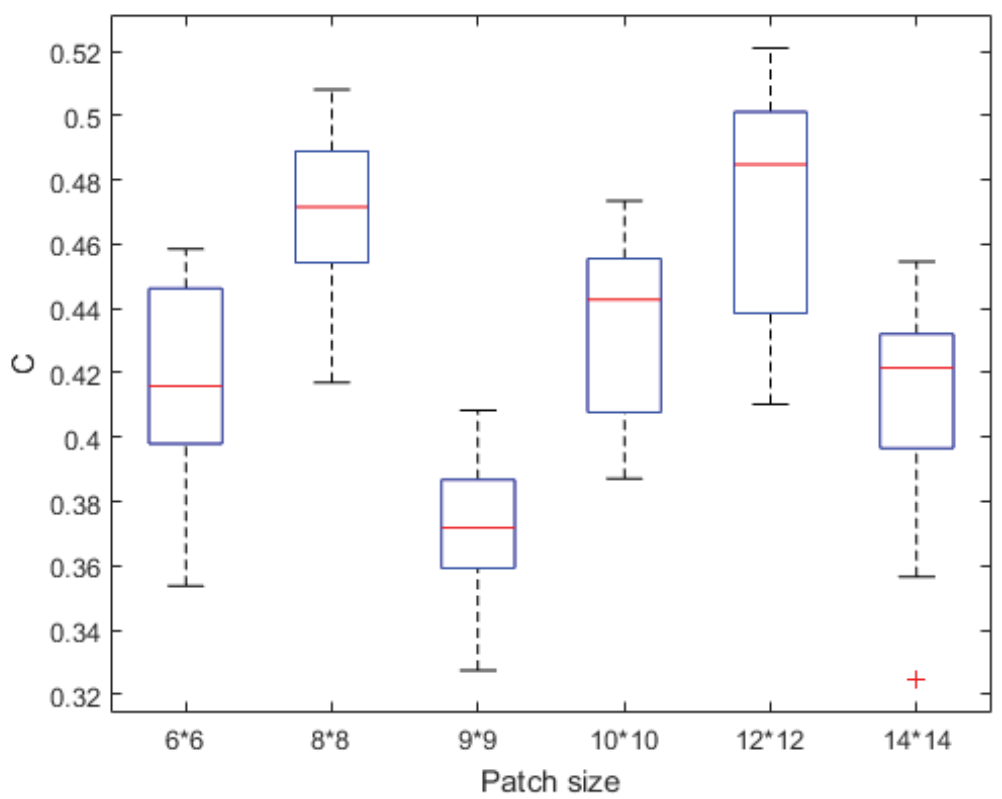

Fig. 4. Values of $C$ with different patch sizes (DRIVE testing set)

of the dictionary patch size is related to the retinal vascular local geometry, and it leads to an imperfect enhancement performance when the patch size isn't suitable for the retinal vascular width. However, the influence of the retinal vascular local geometry can be reduced to a minimum due to the dictionary patch selection and sparse coding. 


\section{ACCEPTED MANUSCRIPT}

\subsection{Effect of dictionary patch selection $(t)$}

Besides the above experiments, the effect of dictionary patch selection $(t)$ was also provided.

Fig. 5 shows the $C$ values of DRIVE testing set with different patch selection threshold values ( $t=5,10,14,18,25$ ), and the patch size is $8 \times 8$. It can be noticed that the average value of $C$ is

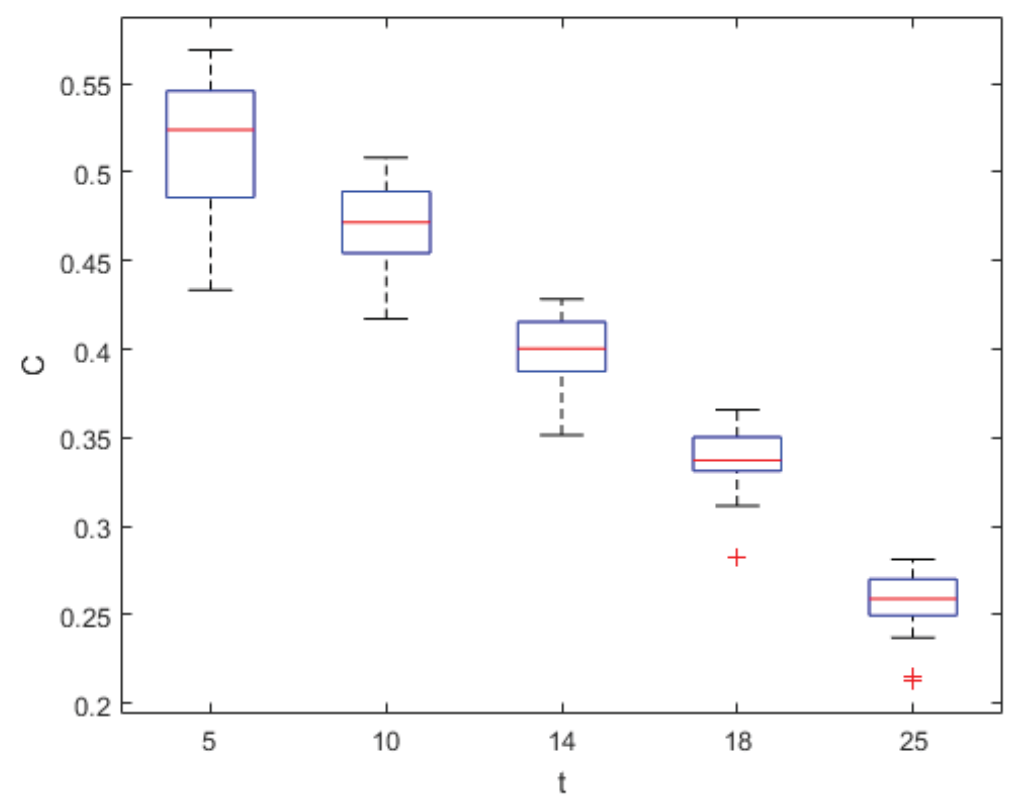

Fig. 5. Values of $C$ with different patch selection threshold values $t$ (DRIVE testing set)

decreased and the mean gray value of the enhanced retinal image is increased with the increase of $t$. The reason of these phenomena is the patches selection during the dictionary building. When the $t$ is increased, the extracted patches from Eq. (1) contain more retinal vessel pixels, so the average value of the ED is increased. Then the average gray value of the enhanced retinal vessel image is also increased, leading to a low contrast between the background and retinal vessel region.

\subsection{Enhancement results}

All the RD and ED used in the retinal vessel enhancement of two databases (DRIVE and 


\section{ACCEPTED MANUSCRIPT}

STARE) were extracted from DRIVE database. We utilized the DRIVE testing set (image index

No.1-20) and STARE as the evaluating data. Fig. 6 and Fig. 7 present some retinal vessel

enhancement results by using different methods. It can be seen that the retinal vascular structures

enhanced by the proposed method are clear and complete in Fig. 6, and the small retinal vessels

are also enhanced efficiently. The $C$ and $C I I$ in Fig. 7 are the corresponding values of Fig. 6.

Obviously, the proposed method has the largest $C$ and $C I I$ values.

a
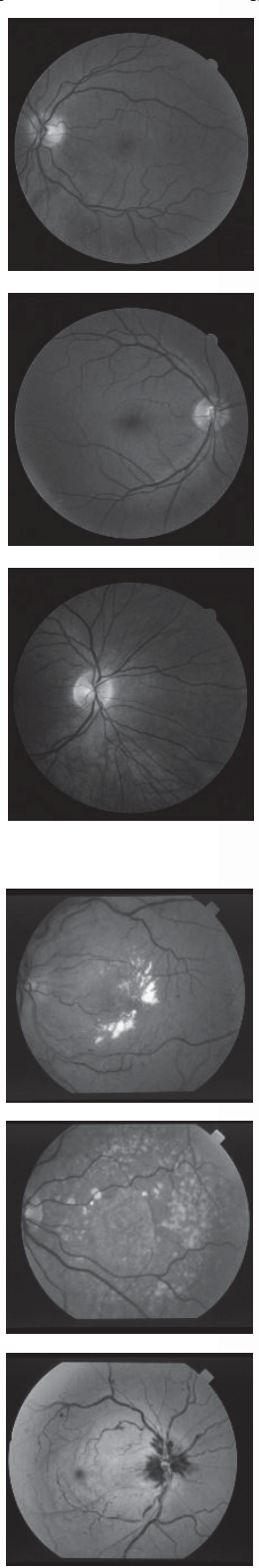
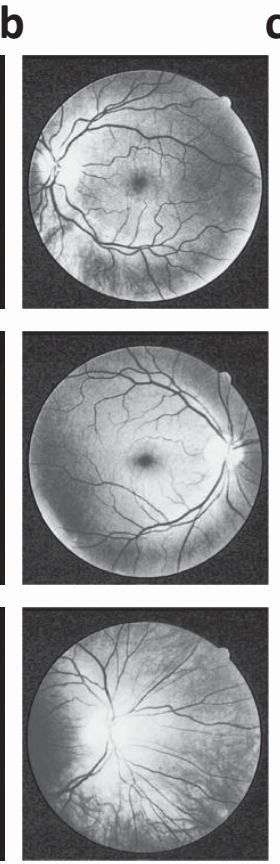

DRIVE testing set, column (a) represents the green channel of the original retinal image
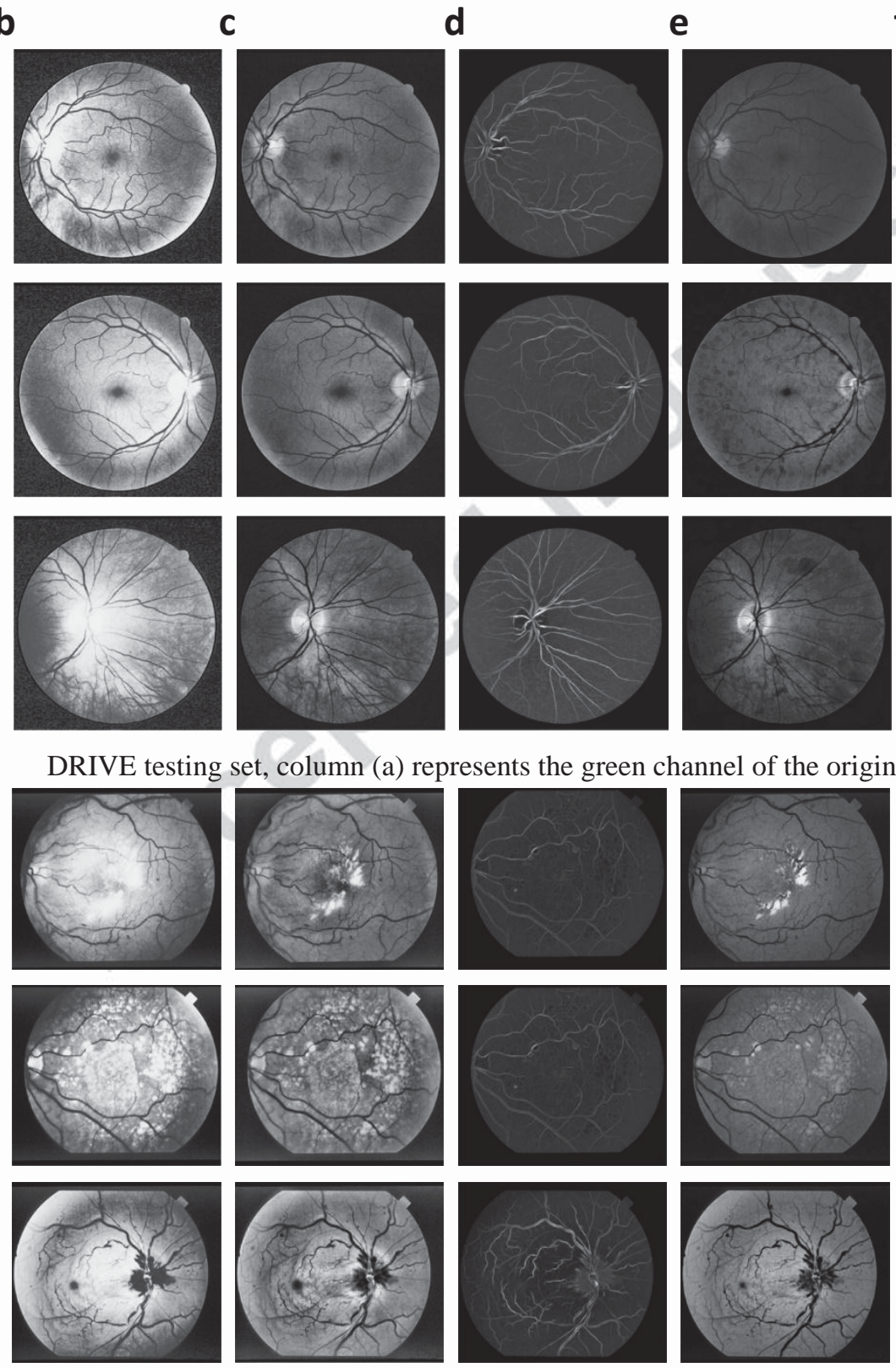
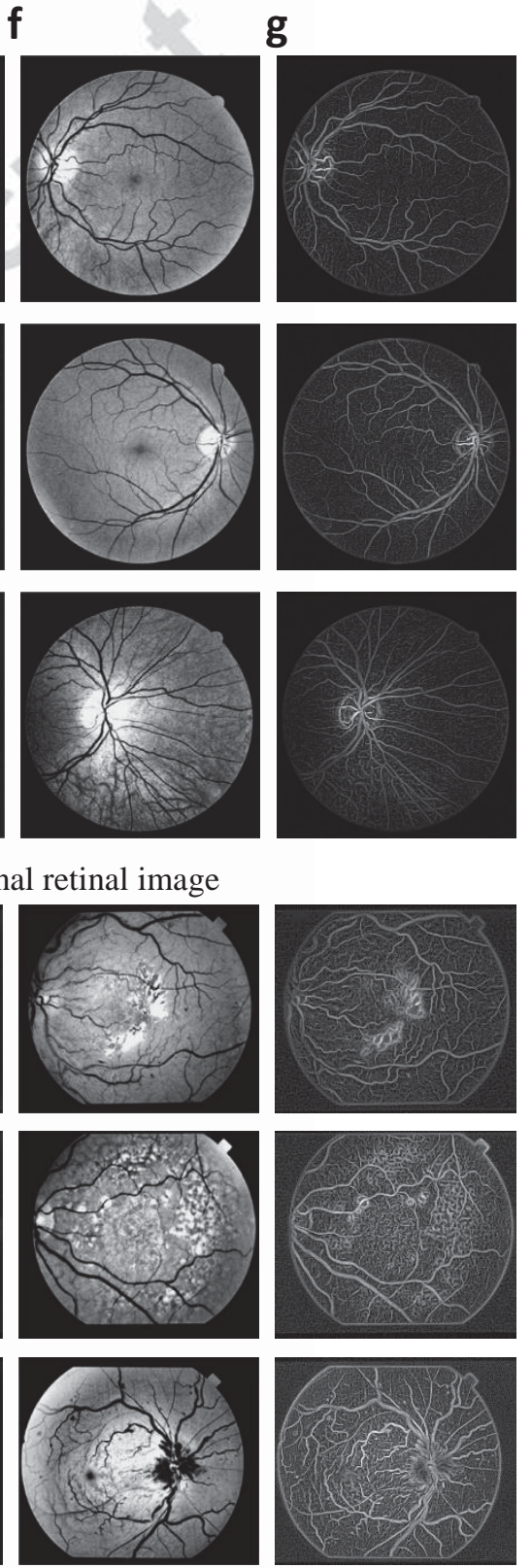


\section{ACCEPTED MANUSCRIPT}

STARE database, column (a) represents the green channel of the original retinal image

Fig. 6. Enhanced results with HE [15], CLAHE [16], methods in [28, 29, 22] and proposed method, column (b) to (g) respectively.

a

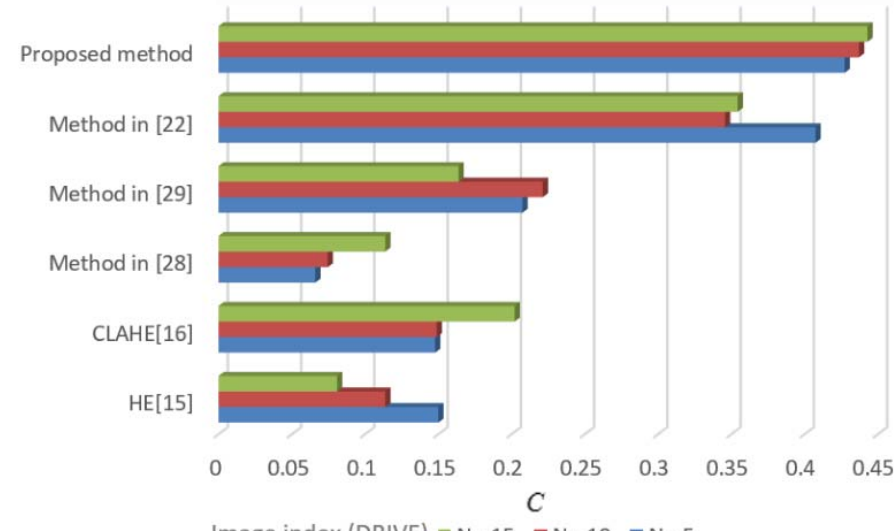

C

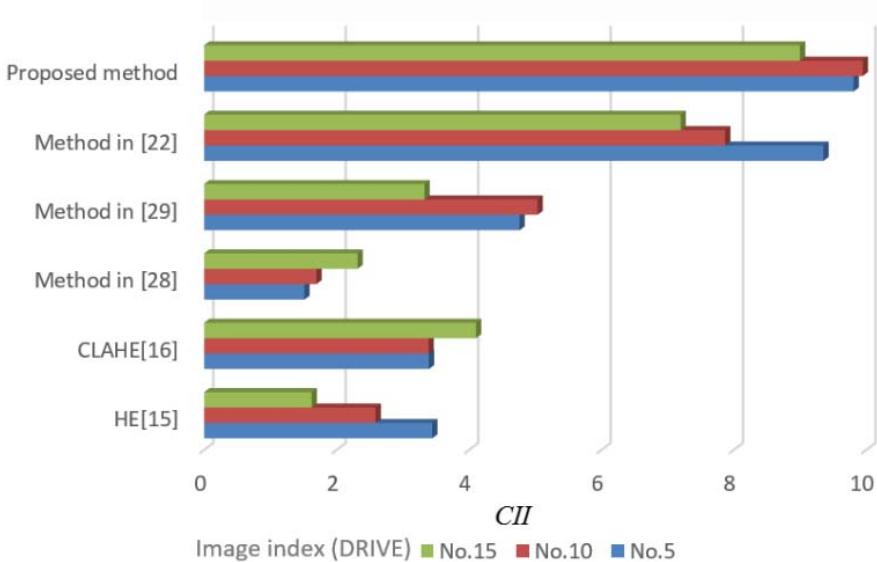

b

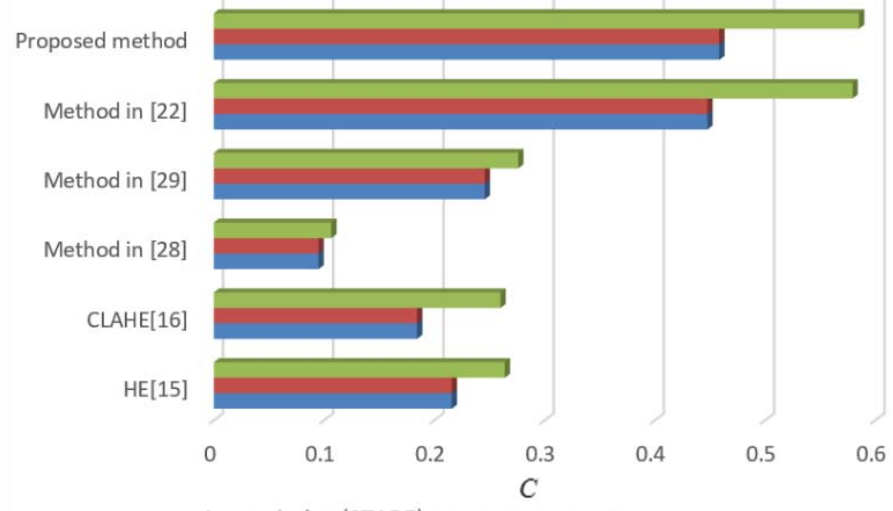

d

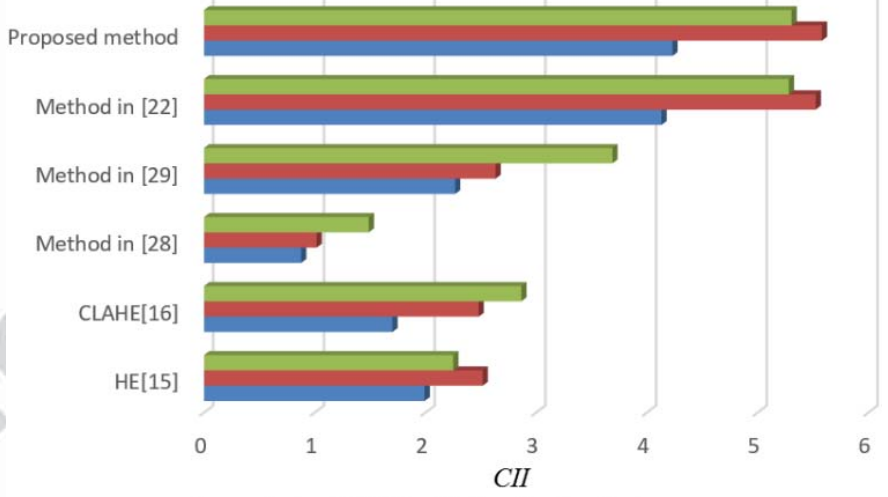

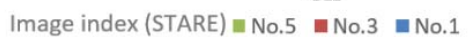

Fig. 7. Comparisons of $C$ and $C I I$ values with different methods in Fig. 6, the image index is corresponding. (a) $C$ values of image index No. 5, 10, 15, in DRIVE testing set, (b) $C$ values of image index No. 1, 3, 5, in STARE database, (c) CII values of image index No. 5, 10, 15, in DRIVE testing set, (d) CII values of image index No. 1, 3, 5, in STARE database.

A comparison of CII values with other enhancement methods in DRIVE testing set (image index No.1- 20) and STARE database (image index No.1- 20) is depicted in Fig. 8. It can be observed that the proposed method has the best performance. For the DRIVE testing data (Fig. 8 


\section{ACCEPTED MANUSCRIPT}

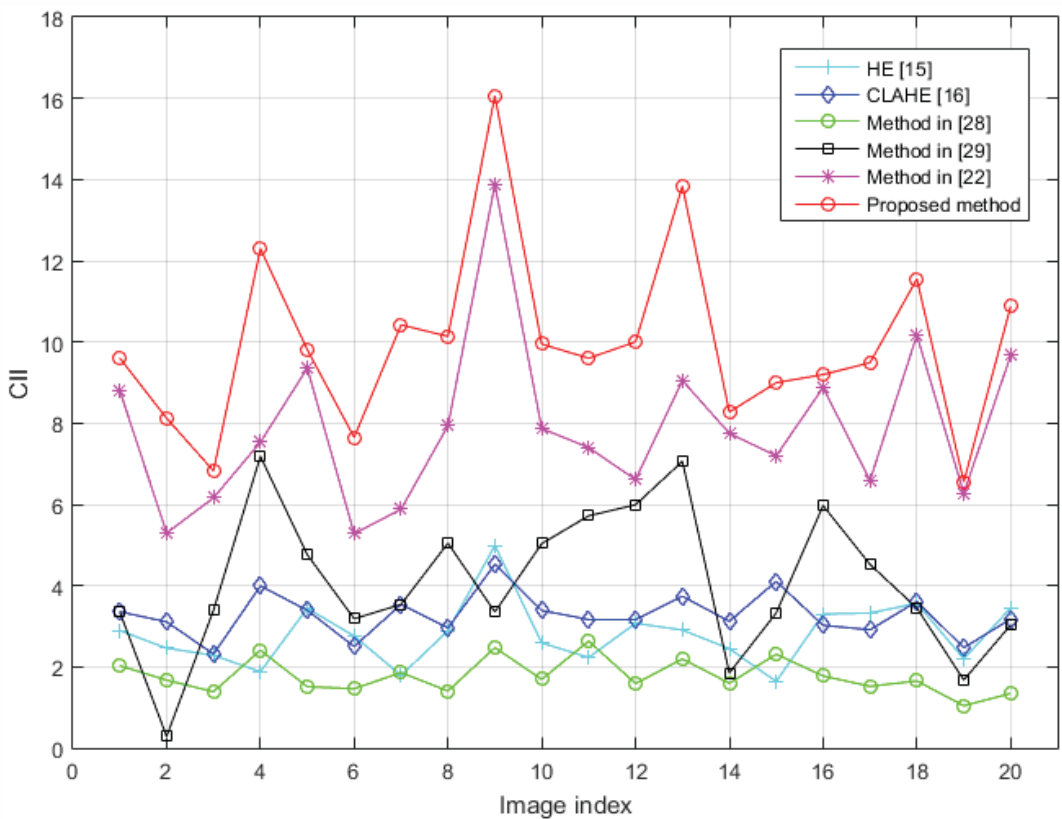

b

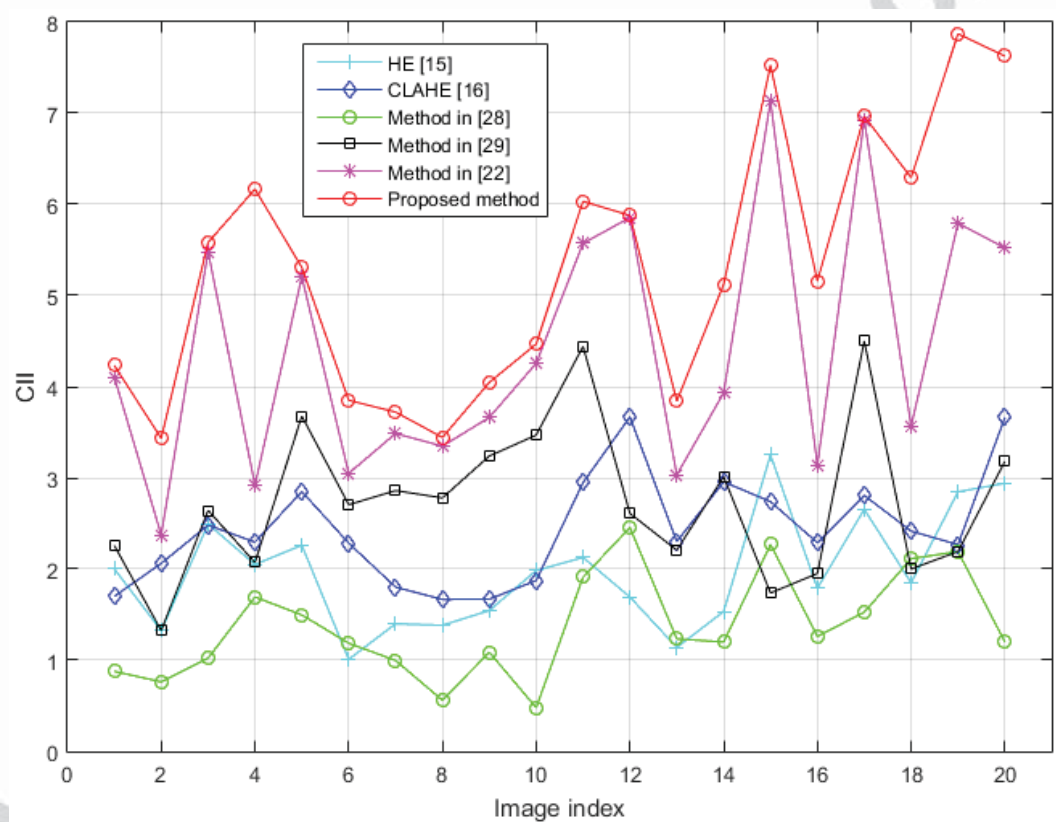

Fig. 8. Values of CII with different methods. (a) DRIVE testing set, (b) STARE database.

(a)), the maximum difference value of CII between the proposed method and method in [22] is

4.7937 (image No.13), and the mean difference value of CII is 2.0842. For the STARE (Fig.

8(b)), the maximum difference value is 3.2480 (image No.4), and the mean difference value of

CII is 0.9124 . 
Compared with HE [15], CLAHE [16], method in [22, 28, 29], the enhanced retinal vessels by the proposed method VE-MSC are much easier to recognize. Based on the above results, it can be concluded that the proposed method has a much better enhancement performance on image contrast, retinal vascular structures, and retinal vascular details. The superiority of our method is mainly contributed to the adoption of multi-dictionary and sparse coding.

\section{Conclusion}

A novel vessel enhancement method (VE-MSC) via multi-dictionary (RD and ED) and sparse coding has been proposed in this paper. We evaluated the proposed method for the retinal vessel enhancement on the DRIVE and STARE databases. Experimental results show that the VE-MSC not only can effectively improve the image contrast but also enhance the retinal vascular structures and details. In the proposed method, two corresponding overcomplete dictionaries (RD and ED) are developed via a patch selection. The RD is utilized to gain the sparse coefficients ( $\alpha$ ), then the enhanced blood vessel image is reconstructed by $\alpha$ and ED. The patch size $(h)$ and patch selection threshold value $(t)$ both affect the enhancement performance of the blood vessel image. Further work includes: (i) improving the enhancement performance with a larger database; (ii) applying on 3D blood vessel enhancement problems; (iii) extending to other image enhancement problems.

\section{Acknowledgments}

This research was supported by the National Natural Science Foundation under grants (81370040).

\section{Reference}


[1] M. Sofka, C. V. Stewart, Retinal vessel centerline extraction using multiscale matched filters, confidence and edge measures, IEEE Transactions on Medical Imaging, 25(12) (2006) 1531-1546.

[2] M. K. Ikram, J. C. M. Witteman, J. R. Vingerling, et al., Retinal vessel diameters and risk of Hypertension the rotterdam study, Hypertension, 47(2) (2006) 189-194.

[3] K. McGeechan, G. Liew, P. Macaskill, et al., Meta-analysis: retinal vessel caliber and risk for coronary heart disease, Annals of internal medicine, 151(6) (2009) 404-413.

[4] M. E. Gegúndez-Arias, A. Aquino, J. M. Bravo, et al., A function for quality evaluation of retinal vessel segmentations, IEEE Transactions on Medical Imaging, 31(2) (2012) 231-239.

[5] T. Y. Wong, R. McIntosh, Hypertensive re-tinopathy signs as risk indicators of cardiovascular morbidity and mortality, British Medical Bulletin, 73(1) (2005) 57-70.

[6] E. J. Sussman, W. G. Tsiaras, K. A. Soper, Diagnosis of diabetic eye disease, The Journal of the American Medical Association, 247(23) (1982) 3231-3234.

[7] M. U. Akram, A. Atzaz, S. F. Aneeque, et al., Blood vessel enhancement and segmentation using wavelet transform, in: IEEE 2009 International Conference on Digital Image Processing, (2009) 34-38.

[8] P. T. H. Truc, M. A. U. Khan, Y. K. Lee, et al., Vessel enhancement filter using directional filter bank, Computer Vision and Image Understanding, 113(1) (2009) 101-112.

[9] E. Ricci, R. Perfetti, Retinal blood vessel segmentation using line operators and support vector classification, IEEE Transactions on Medical Imaging, 26(10) (2007) 1357-1365.

[10] C. Zuo, Q. Chen, X. Sui, Range limited bi-histogram equalization for image contrast enhancement, Optik-International Journal for Light and Electron Optics, 124(5) (2013) 425-431.

[11] S D. Chen, A new image quality measure for assessment of histogram equalization-based contrast enhancement techniques, Digital Signal Processing, 22(4) (2012) 640-647.

[12] T. Celik, Two-dimensional histogram equalization and contrast enhancement, Pattern Recognition, 45(10) (2012) 3810-3824.

[13] J. A. Stark, Adaptive image contrast enhancement using generalizations of histogram equalization, IEEE Transactions on Image Processing, 9(5) (2000) 889-896.

[14] D. Zhang, W. J. Park, S. J. Lee, et al., Histogram partition based gamma correction for image contrast enhancement, in: IEEE 16th International Symposium on Consumer Electronics, (2012) 1-4.

[15] K. Q. Huang, Q. Wang, Z. Wu, Natural color image enhancement and evaluation algorithm based on human visual system, Computer Vision and Image Understanding, 103(1) (2006) 52-63.

[16] A. M. Reza, Realization of the contrast limited adaptive histogram equalization (CLAHE) for real-time image enhancement, Journal of VLSI signal processing systems for signal, image and video technology, 38(1) (2004) 35-44.

[17] K. Singh, R. Kapoor, Image enhancement using exposure based sub image histogram equalization, Pattern Recognition Letters, 36 (2014) 10-14.

[18] E. Lee, S. Kim, W. Kang, et al., Contrast enhancement using dominant brightness level analysis and adaptive intensity transformation for remote sensing images, IEEE Geoscience 
and Remote Sensing Letters, 10(1) (2013) 62-66.

[19] Y. Zhang, L. Wu, S. Wang, et al., Color image enhancement based on HVS and PCNN, Science China Information Sciences, 53(10) (2010) 1963-1976.

[20] S. S. Agaian, K. Panetta, A. M. Grigoryan, Transform-based image enhancement algorithms with performance measure, IEEE Transactions on Image Processing, 10(3) (2001) 367-382.

[21] Y. Li, J. Hu, Y. Jia, Automatic SAR image enhancement based on nonsubsampled contourlet transform and memetic algorithm, Neurocomputing, 134 (2014) 70-78.

[22] M. Liao, Y. Q. Zhao, X. H. Wang, et al., Retinal vessel enhancement based on multi-scale top-hat transformation and histogram fitting stretching, Optics \& Laser Technology, 58 (2014) 56-62.

[23] X. M. Li, L. Li, X. Y. Li, A novel method of image enhancement based on the fractional Fourier transform, in: IEEE 7th International Congress on Image and Signal Processing, (2014) 148-152.

[24] A. Rubel, V. Lukin, M. Uss, et al., Efficiency of texture image enhancement by DCT-based filtering, Neurocomputing, 175 (2016) 948-965.

[25] M. J. Seow, V. K. Asari, Ratio rule and homomorphic filter for enhancement of digital colour image, Neurocomputing, 69(7) (2006) 954-958.

[26] J. S. Paul, J. J. Mathew, C. Kesavadas, MR image enhancement using an extended neighborhood filter, Journal of Visual Communication and Image Representation, 25(7) (2014) 1604-1615.

[27] L. Remaki, M. Cheriet, Numerical schemes of shock filter models for image enhancement and restoration, Journal of Mathematical Imaging and Vision, 18(2) (2003) 129-143.

[28] M. M. Fraz, P. Remagnino, A. Hoppe, et al., An ensemble classification-based approach applied to retinal blood vessel segmentation, IEEE Transactions on Biomedical Engineering, 59(9) (2012) 2538-2548.

[29] J. Oh, H. Hwang, Feature enhancement of medical images using morphology-based homomorphic filter and differential evolution algorithm, International Journal of Control, Automation and Systems, 8(4) (2010) 857-861.

[30] M. Elad, M. Aharon, Image denoising via sparse and redundant representations over learned dictionaries, IEEE Transactions on Image Processing, 15(12) (2006) 3736-3745.

[31] J. Shen, Y. Du, W. Wang, et al., Lazy random walks for superpixel segmentation, IEEE Transactions on Image Processing, 23(4) (2014) 1451-1462.

[32] Y. Zhang, S. Wang, P. Phillips, et al., Binary PSO with mutation operator for feature selection using decision tree applied to spam detection, Knowledge-Based Systems, 64 (2014) 22-31.

[33] Y. Chen, J. Yang, Q. Cao, et al., Curve-like structure extraction using minimal path propagation with back-tracing, IEEE Transactions on image processing, 25(2) (2016) 988-1003.

[34] Y. Chen, L. Shi, Q. Feng, et al., Artifact suppressed dictionary learning for low-dose CT image processing, IEEE Transactions on Medical Imaging, 33(12) (2014) 2271-2292.

[35] S. Li, L. Fang, H. Yin, An efficient dictionary learning algorithm and its application to 3-D medical image denoising, IEEE Transactions on Biomedical Engineering, 59(2) (2012) 
417-427.

[36] R. Chartrand, V. Staneva, Restricted isometry properties and nonconvex compressive sensing, Inverse Problems, 24(3) (2008) 1-14.

[37] R. Baraniuk, M. Davenport, R. DeVore, et al., A simple proof of the restricted isometry property for random matrices, Constructive Approximation, 28(3) (2008) 253-263.

[38] T. Tong, R. Wolz, P. Coupé, et al., Segmentation of MR images via discriminative dictionary learning and sparse coding: Application to hippocampus labeling, NeuroImage, 76 (2013) 11-23.

[39] J. Wright, A. Y. Yang, A. Ganesh, et al., Robust face recognition via sparse representation, IEEE Transactions on Pattern Analysis and Machine Intelligence, 31(2) (2009) 210-227.

[40] B. Ma, J. Shen, Y. Liu, et al., Visual tracking using strong classifier and structural local sparse descriptors, IEEE Transactions on Multimedia, 17(10) (2015) 1818-1828.

[41] M. A. Figueiredo, R. D. Nowak, S. J. Wright, Gradient projection for sparse reconstruction: Application to compressed sensing and other inverse problems, IEEE Journal of Selected Topics in Signal Processing, 1(4) (2007) 586-597.

[42] R. Rubinstein, M. Zibulevsky, M. Elad, Efficient implementation of the K-SVD algorithm using batch orthogonal matching pursuit, CS Technion, 40(8) (2008) 1-15.

\section{Vitae}

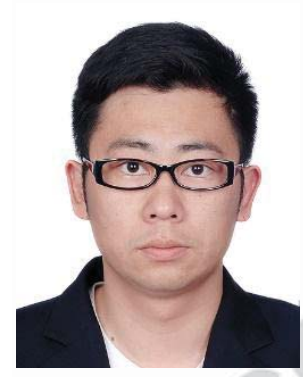

Bin Chen was born in 1987. He received the M.S. degree in pattern recognition from Taiyuan

University of Science and Technology, China, in 2010. He is currently pursuing the Ph.D. degree in School of Computer Science and Engineering, Southeast University. His research interests include image analysis and pattern recognition. 


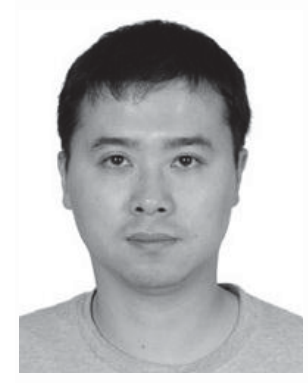

Yang Chen received the M.S. and Ph.D. degrees in biomedical engineering from First Military Medical University, China, in 2004 and 2007, respectively. Since 2008, he has been a Faculty Member with the Department of Computer Science and Engineering, Southeast University, China. His recent work concentrates on the medical image reconstruction, image analysis, pattern recognition, and computerized-aid diagnosis.

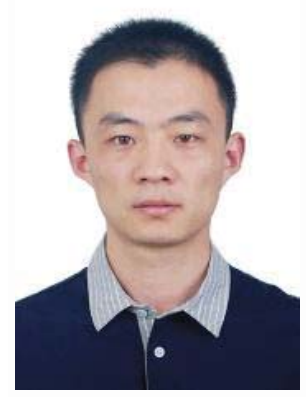

Zhuhong Shao received the B.S. degree in Biomedical Engineering in 2009 from Jilin Medical University, the M.S. degree in Electrical Engineering in 2011 from Beijing Jiaotong University, and the Ph.D. degree in Computer Science and Technology in 2015 from Southeast University. Now he is a lecturer in the College of Information Engineering, Capital Normal University, China. His research interest includes image analysis and pattern recognition. 


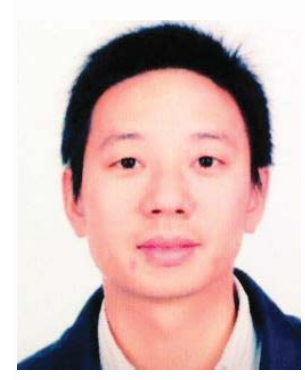

Tong Tong received his B.S. and M.S. degree in biomedical engineering from Beijing Institute of Technology and University of Science and Technology of China respectively. In 2014, he got a Ph.D. degree in computing from Imperial College London. He is now a research fellow in Athinoula A. Martinos Center for Biomedical Imaging, Massachusetts General Hospital / Harvard Medical School, working on brain image analysis.

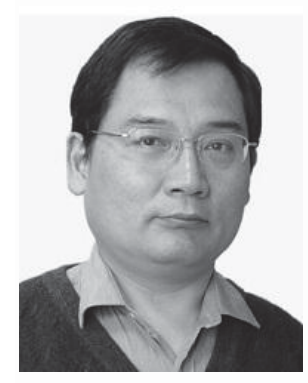

Limin Luo received the Ph.D. degree from the University of Rennes, Rennes, France, in 1986.

He is currently a Professor with the Department of Computer Science and Engineering, Southeast University, Nanjing, China. His current research interests include medical imaging, image analysis, computer-assisted systems for diagnosis and therapy in medicine, and computer vision. 